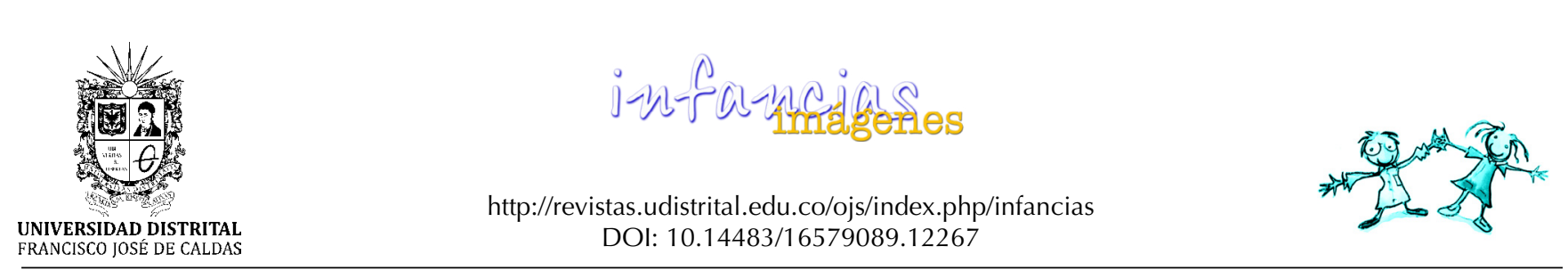

\title{
Cuerpo y movimiento en la educación inicial: concepciones, intenciones y prácticas*
}

\author{
Body and Movement in Early Education: Conceptions, Intentions and Practices \\ Julieth Alejandra Duarte ${ }^{1}$ Laura Carolina Rodríguez ${ }^{2}$ Jaime Alberto Castro-Martínez ${ }^{3}$
}

Para citar este artículo: Duarte, J. A.; Rodríguez, L. C.; Recibido: 01-julio-2017 / Aprobado: 09-septiembre-2017

Castro, J. A. (2017). Cuerpo y movimiento en la educación inicial: concepciones, intenciones y prácticas. Infancias Imágenes, 16(2), 204-215.

\section{Resumen}

La presente investigación se basa en teorías del desarrollo y tiene como objetivo revelar cuáles son las concepciones, intenciones y prácticas de maestros y maestras sobre el cuerpo y el movimiento para potencializar el desarrollo integral de niños y niñas en la educación inicial. En este estudio cualitativo participaron cuatro profesoras de dos jardines de Bogotá, Colombia. Los datos fueron recogidos a través de observaciones y entrevistas. Se concluye que existe una carencia en la capacidad de integrar todas las dimensiones del desarrollo, debido a que las maestras se enfocan en componentes individuales, como la motricidad y las funciones cognitivas, por lo que se resta importancia a otros aspectos del desarrollo como las competencias sociales o la relación con el medio.

Palabras clave: desarrollo del niño; desarrollo motor; educación de la primera infancia; percepción.

\begin{abstract}
This research is based on developmental theories and aims to reveal the conceptions, intentions and practices of teachers on the body and the movement to potentiate the integral development of children in Initial education. In this qualitative study, four teachers participated from two different day-cares in Bogota, Colombia. The data was recorded through observations and interviews. It is concluded that there is a lack of capacity to integrate all the dimensions of development, since teachers focus on individual components such as motor and cognitive functions, thus undermining other aspects of development, such as social skills or their relationship with the environment.
\end{abstract}

Keywords: child development; early childhood education; motor development; perception.

\footnotetext{
* Este artículo es resultado de la investigación Concepciones, intenciones y prácticas docentes que usan el cuerpo y el movimiento para potencializar el desarrollo integral de los niños en la Educación Inicial, de la Universidad del Rosario. La fecha de inicio fue 05-08-2016 y la fecha de finalización 11-04-2017.

1 Psicóloga, Universidad del Rosario (Bogotá, Colombia). Correo electrónico: julieth.duarte@urosario.edu.co

2 Psicóloga en espera de grado, Universidad del Rosario (Bogotá, Colombia). Correo electrónico: rodriguezs.laura@urosario.edu.co

3 Psicólogo, Institución Universitaria Politécnico Grancolombiano (Bogotá, Colombia). Correo electrónico: jcastrom@poligran.edu.co. Autor para correspondencia.
} 


\section{Introducción}

En la actualidad, la educación inicial (El) promueve la formación a través del cuerpo y del movimiento en currículos escolares que incluyen la teoría psicomotriz (Bolaños, 2006), la cual es considerada como uno de los sistemas psicoeducativos que más interés ha despertado en las últimas décadas. Según esta teoría, por medio del cuerpo y el movimiento se favorece el desarrollo integral de los niños, se respeta su globalidad del ser, se fomenta la adquisición de la conciencia de sí y se incentiva su contacto con los demás y con el entorno (Justo, 2014).

Debido a que el cuerpo y el movimiento son concebidos como fundamentales para el desarrollo de los niños y las niñas menores de 6 años, estos elementos se han incorporado en las políticas de los países latinoamericanos (Cordero, 2004), específicamente en los currículos educativos correspondientes a cada país (Centro Internacional de Educación y Desarrollo Humano, 2001).

Por ejemplo, dentro del currículo de El de Chile se indican prácticas que implican la exploración del medio a través del cuerpo y del movimiento, al igual que temáticas de ritmo, equilibrio, sensopercepción y representaciones simbólicas (Blanco, 2005). Así mismo, en Argentina se encuentra que el movimiento hace parte de la enseñanza en la formación inicial, haciendo hincapié en la expresión corporal, para que los niños comuniquen sus emociones, ideas y estados de ánimo (Jaritonsky, 2001). También, se encontró que el currículo de la El de México busca generar experiencias de aprendizaje a través del cuerpo y movimiento, porque resalta el desarrollo de la percepción de sí mismo y de la imagen corporal de los niños y las niñas a través de prácticas como el juego, la danza no estructuralizada y el teatro (Sarle, Ivaldi y Hernández, 2014). Adicionalmente, se encuentra que en Brasil la El considera al movimiento como un lenguaje peculiar de los infantes, mediante el cual los niños y las niñas se comunican, producen experiencias y se constituyen como individuo (Cruz de Oliveira, 2014).

En cuanto al contexto educativo colombiano, se destaca que desde el año 2000 la El comienza a cobrar mayor importancia a nivel estatal. De tal manera que empezaron a desarrollarse políticas, documentos oficiales y currículos que reconocen al cuerpo y a su movimiento como aspectos importantes que influyen en el desarrollo y en el proceso de educación de los niños y las niñas menores de 6 años (Secretaría Distrital de Inclusión Social [SDIS]; Universidad Pedagógica Nacional [UPN]; Secretaría de Educación Distrital [SED], 2013). Actualmente, las orientaciones curriculares para la El proponen cuatro experiencias pedagógicas, reconocidas con el nombre de actividades rectoras: el juego, el arte, la literatura y la exploración del medio. El objetivo principal de estas actividades es encontrar formas dinámicas que satisfagan las necesidades y cubran las características de los niños y las niñas, para que así puedan acercarse a su mundo individual y social potenciando su desarrollo (Ministerio de Educación Nacional [MEN], 2014c).

De esta forma, el desarrollo infantil es considerado un proceso que genera cambios en el comportamiento, los cuales son influenciados por el entorno y la cultura. Este proceso tiene un carácter integral, ya que está constituido por un ámbito afectivo, social, cognoscitivo y psicomotor (Gil et al., 2008). Cada una de estas dimensiones está en constante interacción, por lo que el mejoramiento de un ámbito tendrá un impacto positivo en otras áreas (Bolaños, 2006). Específicamente, el ámbito psicomotor hace referencia a los movimientos corporales, junto a su concientización y control (Gil et al., 2008), poniendo de manifiesto cómo el cuerpo humano es una dimensión esencial de las personas y es considerado la base del desarrollo (Amar, AbeIlo y Tirado, 2004).

\section{Dimensiones de análisis del cuerpo y el movimiento}

Para entender de manera más clara la relación y la influencia del cuerpo y el movimiento en las distintas áreas del desarrollo integral de los niños, se tomó como referencia la investigación sobre la naturaleza de la personalidad realizada por Caspi y Ben (1990, citada por Cano, Rodríguez, García y Antuña, 2005), en donde se distinguen tres tipos de interacciones entre influencias personales y ambientales: las reactivas, en donde la persona actuará ante él o ella en función de su personalidad; las evocativas, en donde los patrones de conducta 
de las personas evocan determinadas respuestas en otros individuos; y las proactivas, en donde la persona hace selección de aquellos ambientes en las que él o ella prefieren estar. A partir de lo mencionado, se definieron tres dimensiones, con sus respectivas familias y categorías, con el fin de facilitar la comprensión y el análisis del cuerpo y el movimiento en relación con el desarrollo infantil: 1) el cuerpo y el movimiento en relación hacia sí mismo, 2) el cuerpo y el movimiento en relación hacia los demás; y 3) el cuerpo y el movimiento en relación hacia el entorno. Sin embargo, es importante aclarar que, pese a la distinción realizada, se reconoce que las tres dimensiones se relacionan entre sí, obteniendo con ello una mirada integral del cuerpo y del movimiento.

\section{Primera dimensión: el cuerpo y el mo- vimiento en relación hacia sí mismo}

\section{Esquema corporal}

Una de las familias más grandes que se encuentra

dentro de esta dimensión es aquella que hace referencia al esquema corporal, es decir, a las representaciones mentales que el niño tiene sobre su propio cuerpo. Palau (2004) menciona que las transformaciones psicomotoras que se dan en la primera infancia fomentan la representación que cada niño y niña va interiorizando sobre su propio cuerpo; algo que es de gran importancia para el desarrollo infantil. De tal manera, se encuentran, dentro de esta familia, las categorías de autopercepción y de consciencia corporal. La primera se entiende como el proceso mediante el cual las personas perciben su cuerpo en relación con el efecto que tiene la variación del tamaño del espacio, y sobre el tamaño percibido de las partes del cuerpo (Gallego del Castillo, 2010). La segunda, hace referencia a la comprensión que se da en los niños y las niñas sobre las propias posibilidades de movimiento corporal, el cual empieza con la identificación de las partes del cuerpo.

\section{Construcción de la identidad}

Otra familia que hace parte de la primera dimensión es aquella que relaciona el cuerpo y el movimiento con la construcción de identidad. Dentro de esta, se hallan distintas categorías: diferenciarse de los demás (Grasso, 2005), confianza en sí mismo
(SDIS, UPN y SED, 2013) y conocimiento de las propias capacidades y personalidad (Ruano, Learreta y Barriopedro, 2006).

\section{Socioafectivo}

El campo socioafectivo es otra familia que se identifica dentro de la primera dimensión, en donde se encuentra que el cuerpo y el movimiento fomentan en los infantes la reafirmación del autoconcepto y de la autoestima (Bolaños, 2006), así como el conocimiento y la autorregulación de sus emociones a través de actividades diarias que requieren trabajo con otras personas (Barbero, 2005).

\section{Funciones cognitivas}

Lení y Wey (2015) señalan que el cuerpo y el movimiento de los niños ayudan a mejorar la atención, memoria, concentración y todas aquellas actividades cognitivas clasificadas como superiores. Esta estrecha relación se ve expresada en los retos diarios que enfrentan los niños y las niñas, los cuales requieren de soluciones que involucran el cuerpo y su movimiento. Así mismo, el desarrollo de la motricidad de los niños y niñas es un dominio importante que permite la adquisición, mejora continua y consolidación de competencias que permiten la obtención de nuevos aprendizajes (Blanco, 2005).

\section{Exploración del propio cuerpo}

Esta familia contiene conceptos como el equilibrio, la coordinación y el control corporal, los cuales se desarrollan a través de las vivencias cotidianas de los niños y las niñas, permitiéndoles adquirir habilidades motrices como saltar y correr. Cuando los niños y las niñas han logrado un mejor dominio corporal es pertinente provocarlos a nuevos retos motrices para que fortalezcan su control corporal y para que descubran posibilidades que aportan elementos en su desarrollo psicomotor, tales como actividades que impliquen búsqueda, persecución, caídas, rodar, hacer volteretas, etc. (SDIS, UPN y SED, 2013).

\section{Segunda dimensión: el cuerpo y el mo- vimiento en relación con los demás}

Competencias sociales

Dentro de esta familia se encuentra la categoría de relaciones sociales, pues el cuerpo y el movimiento 
posibilita a los infantes realizar un contacto con las personas que les rodean, lo que les brinda retroalimentación y les genera un aprendizaje, al hacer una construcción conjunta con su propio sistema social (Bolaños, 2006). Otra categoría que se identifica en esta familia es la de sociedad y cultura, en la cual Remorini (2010) reconoce que el cuerpo y el movimiento se relacionan con la cultura social en la que las personas se desenvuelven, convirtiéndose en un vehículo de expresión de marcadores de identidad, clase, género, edad, etc.

\section{Competencias emocionales}

Acá se encuentra la expresión emocional que se da mediante el cuerpo y el movimiento, porque desde los primeros años de vida los niños y las niñas desarrollan recursos expresivos para comunicar sentimientos, emociones, ideas y estados de ánimo a través de gestos y movimientos (Ruano, Learreta y Barriopedro, 2006). Por otro lado, se encuentra que cuando un infante tiene mayor conciencia sobre sus capacidades motoras es capaz de desarrollar habilidades como la empatía y el reconocimiento de los demás (Bolaños, 2006).

\section{Competencias comunicativas}

Otra familia que hace parte de esta dimensión es la comunicación, debido a que, desde los primeros años de vida, los seres humanos han usado su cuerpo para manifestarse y comunicarse con su entorno y con las personas que los rodean (García, Pérez y Calvo, 2013). Por tal razón, el movimiento, como forma de expresión corporal, es considerado como una de las primeras formas de comunicarse de los niños y las niñas, convirtiéndose así en el lenguaje del cuerpo, el cual le permite comprender el mundo (SDIS, UPN y SED, 2013). También se encuentra que promover en los infantes el movimiento ayuda a potenciar sus capacidades de lenguaje y de lectura (Campo, 2010).

\section{Competencias ciudadanas}

Shoval, Sharir y Shulruf (2014) proponen usar el cuerpo y el movimiento para generar estrategias que fomenten las competencias ciudadanas, argumentando que las actividades que involucran el movimiento corporal les dan la oportunidad a los infantes de cooperar, seguir instrucciones, elegir, participar y aprender en los procesos sociales. Así, dentro de esta familia, aparecen las categorías: cooperación, seguimiento de instrucciones y comprensión de reglas y normas.

\section{Tercera dimensión: el cuerpo y el movi-} miento en relación con el entorno

Una primera categoría que se relaciona con esta familia es la construcción de representaciones sobre el tiempo, la cual se desarrolla a partir del conocimiento que tiene el individuo sobre su propio cuerpo; en donde este debe ser consciente de lo que sucede en su interior al momento de localizarlo dentro de un tiempo determinado (SDIS, UPN y SED, 2013). Otras categorías que se pueden señalar en esta familia son: la representación personal para ocupar el espacio (Cabezas, 2005) y la construcción de representaciones sobre el espacio (Sánchez et al., 2014).

Construcción de conocimiento y aprendizaje Los niños y las niñas, a través de la corporeidad y el movimiento, realizan un contacto con el medio ambiente que les permite retroalimentación, les genera un aprendizaje y aprehenden el conocimiento por medio de los sentidos, al hacer una construcción conjunta con su propio entorno (Bolaños, 2006). Además, se tiene en cuenta que ellos, por naturaleza, son curiosos, exploradores y, en su diario vivir, descubren nuevas cosas por medio del tacto y el movimiento (Fernández, 2010).

Como se puede apreciar, de acuerdo con la literatura, es de resaltar la importancia del cuerpo y del movimiento para el desarrollo integral de los niños y las niñas. Además, el rol de los docentes y sus prácticas, mediante estrategias enfocadas en la corporeidad y el movimiento, son esenciales en la potencialización del desarrollo de la infancia. Sin embargo, esta misma revisión documental evidencia un vacío en este sentido en la El de Colombia, ya que no hay un mayor conocimiento sobre cuáles y cómo son llevadas a cabo las prácticas que implican el cuerpo y movimiento en este escenario, así como tampoco se conocen las concepciones que tienen los maestros sobre el cuerpo y el movimiento en relación con el desarrollo integral de los 
niños. Por lo anterior, los objetivos de la presente investigación son: 1) revelar cuáles son las concepciones que tienen los maestros sobre el cuerpo y el movimiento relacionadas con desarrollo integral de los niños y las niñas; 2) dar cuenta de las prácticas, Ilevadas a cabo por maestros y maestras, que involucran el cuerpo y el movimiento de niños y niñas en jardines infantiles de la ciudad de Bogotá, Colombia; y 3) evidenciar con qué intención estas son llevadas a cabo.

\section{Metodología}

En esta investigación se utilizó el diseño de teoría fundamentada de Strauss y Corbin (2002), el cual es muy útil para comprender procesos educativos y psicológicos, ya que permite identificar los conceptos implicados del fenómeno estudiado y las acciones e interacciones de los participantes involucrados en la investigación. Es importante mencionar que se usó la medida de triangulación de datos, la cual consiste en la utilización de distintas fuentes de recolección, permitiendo así obtener datos con una mayor riqueza, profundidad y amplitud (Hernández, Fernández y Baptista, 2010).

\section{Participantes}

La muestra que participó en esta investigación fue de dos profesoras del grado prejardín y dos del grado jardín, de dos instituciones privadas diferentes de la ciudad de Bogotá, Colombia. Sus edades varían entre 24 a 30 años.

\section{Técnicas de recolección de información \\ Observación no participante}

La observación es un instrumento de tipo cualitativo con la cual el investigador obtiene información que puede ser omitida, ya sea voluntaria o involuntariamente por el investigado (Sarduy, 2007). La intención es que, a través de esta técnica, se identifiquen ciertos elementos que se convertirán en unidades de análisis, de acuerdo con los objetivos de la investigación (Hernández, Fernández y Baptista, 2010). En este caso, se buscó identificar las prácticas comunes que los maestros y maestras realizan en la El, para así analizar qué tanto son involucrados el cuerpo y el movimiento de los niños y las niñas en estas actividades.

\section{Entrevista semiestructurada}

Las entrevistas semiestructuradas son aquellas indagaciones que se basan en una guía de preguntas que permiten recoger la información necesaria según los objetivos de la investigación (Hernández, Fernández y Baptista, 2010). En este caso, las concepciones, creencias y percepciones que tienen los maestros y maestras sobre la importancia del cuerpo y del movimiento para el desarrollo de los infantes.

\section{Procedimiento}

Para llevar a cabo la investigación, inicialmente se obtuvo el consentimiento informado por parte de las maestras, niños y niñas, padres y rectores de las instituciones implicadas en la investigación. En seguida se realizaron las respectivas observaciones, basadas en las dimensiones anteriormente explicadas, las cuales se realizaron en dos jardines. En cada institución se hicieron dos sesiones de observación que duraron aproximadamente 45 minutos: una, en la clase del grado jardín, y la otra, en la clase del grado pre-jardín, obteniendo así un total de cuatro sesiones de observación no participativa. Después, se prosiguió a realizar entrevistas a las maestras que dirigieron las clases observadas, con el fin de recoger información sobre las concepciones que tenían sobre el cuerpo, el movimiento y las intencionalidades de sus prácticas. Por último, se unieron los resultados observados y arrojados para analizarlos a través del programa Atlas.ti versión 7.

\section{Consideraciones éticas}

En primera instancia, para llevar a cabo este trabajo, se envió una propuesta de la metodología de este estudio al comité de ética de la universidad, con el fin de recibir el aval ético y así poder llevar a cabo la investigación. Una vez aprobado, se realizó el proceso de consentimiento con las directoras, docentes y padres de familia de las respectivas instituciones. Por último, se realizó un formato de asentimiento especial para los niños, con el fin de facilitar este proceso, que se llevó a cabo por medio de un diálogo con cada uno.

\section{Resultados}

Los resultados se agruparon de acuerdo con cada objetivo propuesto en la presente investigación: las 
concepciones de las maestras acerca de la importancia del cuerpo y el movimiento para el desarroIlo integral, las prácticas Ilevadas a cabo por las maestras que involucran el cuerpo y el movimiento, y las intenciones de estas al realizar dichas prácticas. Por cada objetivo, se encontrará el análisis de los resultados divididos en las tres dimensiones propuestas: 1) el movimiento hacia sí mismo, 2) el movimiento en relación con los demás, y 3) el movimiento en relación con el entorno.

\section{Resultados de las concepciones}

De manera general, se encontró que para las maestras el cuerpo y el movimiento cumplen una función muy importante en el desarrollo de los niños y las niñas, así como también dentro del contexto de la El. Las maestras que participaron en las entrevistas manifestaron que el cuerpo y el movimiento hacen parte de las actividades diarias y de la vida cotidiana de los niños y las niñas y, por tal razón, estos conceptos pueden ser utilizados como indicadores que permiten observar y tener un indicio de cómo se encuentra el desarrollo del niño y la niña. "Yo considero que el cuerpo y el movimiento son importantes en la educación inicial, porque cuando ellos se mueven e interactúan, aprenden más" $(3: 5)^{4}$.

Por otro lado, las maestras también mencionaron que el cuerpo y el movimiento son un medio para que los niños se sientan motivados en su proceso de aprendizaje, por lo que es percibido como sinónimo de diversión y de libertad.

\section{Primera dimensión}

La familia de exploración del propio cuerpo, perteneciente a esta primera dimensión, es la que más predominó en los hallazgos debido a que las cuatro maestras resaltaron el valor de la motricidad en el

\footnotetext{
${ }^{4}$ Los números que aparecen en el apartado son las citas de las voces de los investigadores y de las participantes. Estos corresponden al análisis realizado a través del programa Atlas.ti. Este programa genera códigos a las citas en las entrevistas, los cuales permiten la ubicación de estas donde se presentan las categorías. El primer número representa el documento primario donde se encuentran las observaciones y las entrevistas, y el segundo número, la línea en la que se encuentra la cita que compone la categoría. Los documentos del 1 al 4 referencian las entrevistas, y los documentos del 5 al 8 las observaciones.
}

desarrollo de los niños, entendiendo al cuerpo y al movimiento como un medio para fomentar las habilidades motrices. "Yo pienso que el cuerpo y el movimiento en la educación inicial son muy importantes, ya que estimulan la motricidad, porque eso es lo que maneja el cuerpo: la motricidad gruesa y la motricidad fina" $(2: 2)$.

De tal manera, hubo una gran participación de las categorías equilibrio, coordinación y control corporal en las concepciones de las maestras. Las otras familias pertenecientes a esta dimensión (esquema corporal y socioafectivo) no fueron predominantes en el análisis de los resultados. Sin embargo, se encontraron vínculos con estas familias en categorías como consciencia corporal (esquema corporal) y autoconcepto, autorregulación y autoestima (socioafectivo).

\section{Segunda dimensión}

Se encontró una mayor evidencia en la familia competencia emocional, en donde las maestras manifestaron que, a través del cuerpo y el movimiento, se puede detectar el estado de ánimo de los niños y las niñas.

Si tu vez a un niño que no realiza nada, que no se expresa, pues uno ya puede ver que está sucediendo algo; pero si el niño está, digamos, activo, es decir, se mueve, todo lo que, digamos, tú le explicas, lo que él coge, y la manera en que lo coge, pues eso ya nos está indicando algo. Entonces, todo eso nos da a nosotras una cierta forma de ver al niño, en qué proceso está, o sea, cómo se siente anímicamente. (1:19)

Así pues, la categoría expresión de estado de ánimo aparece de manera importante en las concepciones de las maestras sobre el cuerpo y el movimiento en concordancia con el desarrollo integral de los niños y niñas. Otra familia que se encuentra dentro de esta dimensión y que obtuvo una mayor participación en relación con las concepciones, fue la de competencia comunicativa, debido a que las maestras manifestaron que la comunicación de los infantes con los demás se da por medio del cuerpo y del movimiento y, a través de estos, expresan sus ideas. Por consiguiente, la categoría expresión de ideas y la subcategoría enriquecimiento de 
vocabulario de la categoría lenguaje, se evidencian en las concepciones de las maestras. "Los niños recuerdan mucho con las rondas, como el color, formas geométricas, vocales y números, cuando uno les muestra una imagen ellos lo asocian muy rápido" (4:11).

\section{Tercera dimensión}

De manera general, se encontró muy poca interacción entre las familias pertenecientes a esta dimensión y las concepciones de las maestras sobre el cuerpo y el movimiento en relación con el desarrollo integral de los niños y las niñas. Únicamente se halló una concordancia con la categoría manipulación, de la familia construcción de sentido del espacio y de la temporalidad, pues se identificó que, para una maestra, a través del cuerpo y el movimiento los niños y las niñas adquieren conocimiento de los objetos por medio de sus sentidos.

\section{Resultado de las prácticas}

210 Primera dimensión

En cuanto a esta dimensión y a las prácticas de las maestras que involucran el cuerpo y el movimiento, no se encontró algún soporte de las familias socioafectivo y construcción de la identidad en las prácticas pedagógicas de las maestras.

En contraparte, al igual que en las concepciones y las intenciones, la familia de exploración del propio cuerpo, perteneciente a esta primera dimensión, es la que más predominó en los hallazgos relacionados con las prácticas de las maestras. Es importante mencionar que las categorías equilibrio, control corporal y coordinación están muy asociadas, debido a que son componentes de la motricidad y, como ya se ha mencionado, la mayoría de prácticas incluían ejercicios que potencian las habilidades motrices. La práctica que más realizan las maestras, y que más se evidenció por medio de las observaciones y las entrevistas, es la de grafomotricidad, en donde los niños y las niñas colorean, rasgan, dibujan, puntean, decoran, pintan, manejan plastilina, etc., muy relacionado con el arte manual. De igual manera, se encontró que otras prácticas como el juego y las rondas musicales también son utilizadas para afianzar la motricidad gruesa y fina en los niños y las niñas.
La maestra les entregó un pedazo de papel silueta de color amarillo y les explicó que había que rasgar el papel en pedacitos y hacer bolitas con los dedos, para así pegarlos en el cono, ella mencionó en voz alta que era importante que las hicieran con las Ilamas de los dedos para manejar la motricidad. (8:14)

Adicional a las tres categorías anteriormente mencionadas, pertenecientes a esta familia, se encontró el concepto de ritmo, que no se había definido en el componente teórico, y que a través del ejercicio del análisis de resultados se encontró la necesidad de incluirlo dentro de esta familia. Dado que fue notorio en las prácticas relacionadas con el cuerpo y el movimiento de las maestras, se estableció como categoría emergente, en donde las rondas musicales sobresalieron en el análisis de las prácticas.

Por otro lado, siguiendo con la familia esquema corporal, se halló una mayor relevancia en las subcategorías pertenecientes a esta familia. Por un lado, se identificaron prácticas como la gimnasia cerebral (que incentivaba a los niños y niñas a que identificaran sus partes del cuerpo o también esto se realizaba a través de dibujos y de rondas musicales); y, por otro, la categoría sensibilidad, la cual se vio reflejada en prácticas manuales que permiten a los niños sentir elementos de distintas texturas.

\section{Segunda dimensión}

Se identificó que, a través de una cantidad importante de prácticas, los niños y las niñas aprenden nuevo vocabulario como números, vocales, colores, figuras, entre otros. Los ejercicios que reflejan lo anteriormente mencionado son: la escritura, la transcripción de letras, la imitación, los juegos, los videos, rondas musicales y actividades que implican la grafomotricidad en las cuales, a través de dibujos, trazos, pintura y decoración desarrollan esta habilidad. Por ende, se evidenció la categoría enriquecimiento de vocabulario, perteneciente a la familia de competencias comunicativas, en las prácticas pedagógicas. Otra categoría perteneciente a esta familia, y la cual se pudo identificar en las prácticas, es la de desarrollo de capacidad de escucha, que se manifiesta, principalmente, a través de rondas musicales, canciones y ejercicios de gimnasia cerebral. 
Se encontró una participación de la categoría relaciones sociales, perteneciente a la familia competencias sociales, en las prácticas de las maestras como aquellas que implican interacción del niño y la niña con sus pares, tales como el juego y las rondas musicales; o actividades como colorear y manipular plastilina, en donde se les da la libertad de conversar con sus compañeros y compañeras. Por último, la categoría de seguimiento de instrucciones, perteneciente a la familia de competencias ciudadanas, fue relevante en las prácticas aplicadas por las maestras de las instituciones; esto se observó en prácticas como rondas musicales y gimnasia cerebral, que exigía el seguimiento de ciertas instrucciones.

\section{Tercera dimensión}

Se encontraron algunos soportes de la categoría de manipulación, perteneciente a la familia construcción de sentido del espacio y de la temporalidad, específicamente en las prácticas de grafomotricidad en donde se manipulan diferentes materiales con sus manos, permitiéndole al niño y a la niña diferenciar distintas texturas y características de distintos objetos.

Por medio de dibujos la maestra les explica los colores y las frutas, ¿son cerezas? ¿Qué son? ¿Son ácidas o son dulces. Los niños lo decoran. Rasgan el papel, lo pegan en la hojita de las cerezas que les entregó la profesora. Después lo decoran con escarcha y con pintura. $(6: 5)$

Por último, la categoría adquisición por medio de los sentidos, perteneciente a la familia de conocimiento y aprendizaje, se vio evidenciada en los juegos en los que se estimula a los niños y niñas a que identifiquen objetos por medio de los sentidos y los clasifiquen según sus características.

\section{Resultados de las intencionalidades}

\section{Primera dimensión}

Con relación a esta dimensión, y a la intencionalidad de involucrar el cuerpo y el movimiento de los niños y las niñas en sus prácticas por parte de las maestras, la familia exploración del propio cuerpo, perteneciente a esta primera dimensión, fue la que más predominó en los hallazgos, ya que se identificó que las prácticas realizadas por ellas iban encaminadas al desarrollo de la motricidad, especialmente la motricidad fina. "En medio de la actividad, la maestra dio la siguiente instrucción: vamos a aprender a manejar el crayón, vamos a empezar a mover los dedos" (8:3).

Al mismo tiempo, se encontró que el afianzar la motricidad en los niños y las niñas tienen distintas intenciones, según la maestra. Por ejemplo, una de ellas expresó enfocarse mucho en la motricidad fina debido a que un requerimiento que le impone el currículo escolar es lograr que los niños salgan de ese curso sabiendo transcribir su nombre $y$, por lo tanto, se les guío a través de muchas actividades que permitan soltar los dedos y mejorar el agarre.

Por otro lado, se encontró que distintas o prácticas, como el juego, o aquellas actividades que impliquen el movimiento de todo el cuerpo se realizan con el fin de afianzar la motricidad gruesa. También, a través de estas, las maestras tienen la intención de observar a quienes tienen dificultades en estas habilidades, para así trabajar en ellas. De tal manera, las categorías equilibrio, coordinación y control corporal, poseen una relación importante con las intenciones de las maestras, al igual que la familia de funciones cognitivas, en donde se destaca el desarrollo de la atención.

En contraparte, no se encontraron soportes de las familias de construcción de la identidad y socioafectivo en las intenciones de las maestras.

\section{Segunda dimensión}

Se encontró que una de las intenciones principales de las cuatro maestras es generar el aprendizaje de números, vocales, figuras geométricas, colores y otros conceptos en los niños y niñas, ya que es un requisito del currículo escolar de la institución, y esto lo realizan a través de distintas prácticas. "Los niños recuerdan mucho con las rondas, como el color, formas geométricas, vocales y números. Cuando uno les muestra una imagen ellos lo asocian muy rápido" (4:11).

Por lo tanto, la subcategoría enriquecimiento de vocabulario de la familia competencias comunicativas, que hace parte de la categoría lenguaje, obtuvo una participación importante en las intenciones de las maestras. 


\section{Tercera dimensión}

En cuanto a esta tercera dimensión, y a las intenciones de las maestras de involucrar el cuerpo y el movimiento de los niños y las niñas en sus prácticas, no se encontraron evidencias de la familia conocimiento y aprendizaje en las intenciones de las maestras. Sin embargo, aunque fue poco, se halló un vínculo con la otra familia perteneciente a esta dimensión: construcción de sentido del espacio y de la temporalidad, en donde se identificó que una maestra no solamente tiene la intención de que los niños y niñas identifiquen las distintas partes de sus cuerpos, sino que relacionen las funciones de cada parte con el entorno en el que se encuentran.

\section{Discusión y conclusiones}

A través de la presente investigación se logró dar respuesta a las preguntas y a los objetivos inicialmente planteados, debido a que el análisis de las observaciones y de las entrevistas realizadas a las cuatro maestras permitieron identificar, de manera

212 general, las concepciones que tienen sobre el cuerpo y el movimiento en relación con el desarrollo integral de los niños y niñas, las prácticas que involucran al cuerpo y el movimiento. A su vez, se identificó con qué propósito estas son llevadas a cabo. Al igual que los resultados, la discusión se presentará según los tres objetivos planteados por la investigación.

\section{Concepciones}

En conclusión, se evidencian dos formas de concebir al cuerpo y el movimiento como potenciadores del desarrollo de los niños por parte de las maestras: la primera es la del cuerpo y el movimiento como elementos importantes para desarrollar las habilidades motrices de los niños y las niñas, la cual sobresalió de manera importante en las narrativas de las maestras; y la segunda hace referencia al cuerpo y el movimiento como el medio de expresión principal de los infantes, de sus ideas y de sus estados de ánimo.

Pese a estas ideas, se observan dos limitaciones: por una parte, la manera como se entiende la motricidad, ya que no se concibe lo motriz como un elemento importante de la corporalidad que permite la construcción del sujeto, sino que se percibe como un factor que fomenta el control corporal. Además, hay poco conocimiento de los beneficios que el desarrollo motriz genera en otras áreas del desarrollo, tales como la estimulación de los músculos de la lengua, que permite el desarrollo del lenguaje (Erikson, 1963, citado en Robles, 2008), o la generación de la autonomía para explorar el mundo y para interactuar con el medio, desarroIlando habilidades y tomando decisiones propias (Bruner, 1970, citado en Lení y Way, 2015).

\section{Prácticas}

En relación con las prácticas educativas que las maestras realizan en la $\mathrm{El}$, donde involucran el cuerpo y el movimiento, se encontró que se recurren a una gran variedad de actividades como los dibujos, el arte, los juegos, gimnasia cerebral, ejercicio físico, rondas musicales y grafomotricidad, donde estas dos últimas son las más relevantes. Por otro lado, es importante mencionar que, para algunas maestras, el realizar prácticas que involucran el cuerpo y el movimiento de los niños y las niñas se relaciona fuertemente con la clase de educación física, situación que suele ser encontrada en estudios similares, como el de Cruz de Oliveira (2014), pues se halló que los profesores no conciben al cuerpo y al movimiento como elementos centrales en el proceso educativo ya que piensan que estos son temas que deben ser tratados especialmente en el deporte o por expertos de educación física.

Aunque se encontraron estas prácticas, existen otras relacionadas con el cuerpo y el movimiento que no se evidenciaron en la presente investigación y que sí hacen parte de las actividades rectoras, tales como la expresión dramática (MEN, 2014a). Esta última es importante ya que fomenta la interacción entre los pares (Castro et al., 2015) y potencializa la comunicación con el otro, a través de distintos movimientos corporales (MEN, 2014a). Este resultado coincide con otros estudios como el de Castro et al. (2015), en donde tampoco se han encontrado evidencias de la utilización, de manera contundente, de esta práctica en las clases de la EI en Colombia.

Es importante recordar que, durante el proceso de análisis de las observaciones y entrevistas, se identificó al ritmo como una categoría emergente, 
debido a que las maestras hacen un uso frecuente de las rondas musicales durante sus clases. El ritmo incluso está en uno de los documento del MEN (2014a) referido al arte, en donde se plantea que la expresión musical debe centrarse en el goce y diversión, así como en la posibilidad de explorar y generar un acercamiento a la lengua materna y a la expresión de emociones (Castro et al., 2015); esto no se vio reflejado en las prácticas de las maestras pues se observó que el uso de las rondas musicales se centró más en la generación de aprendizaje de conceptos y en el fortalecimiento de la motricidad gruesa que en la exploración.

Por último, otra de las prácticas encontradas en esta investigación fue el juego, pilar importante dentro de la política de El de Colombia, debido a que es considerado como una actividad inherente a la edad escolar. Además, permite a los niños y niñas comunicar con su cuerpo distintas maneras de su forma de ser, interactuar con su entorno exterior y sus pares, y expresar su estado de ánimo (MEN, 2014b). No obstante, se evidencia que los maestros realizan los juegos con el fin de fortalecer la motricidad gruesa y generar aprendizaje de conceptos, por ende, el nivel de estructuración de esta actividad es alta, algo que es contrario a la política ya que, según esta, los juegos deben ser espontáneos y no tan guiados (Castro et al., 2015).

\section{Intencionalidad}

Se evidenció que la intención primordial de las maestras es trabajar el cuerpo y el movimiento de los niños y niñas en sus clases. Esto coincide con lo mencionado por Aisenstein y Scharagrodsky (2006), quienes aseguran que, durante las últimas tres décadas, ha aumentado el reconocimiento del rol del cuerpo y del movimiento en la praxis escolar.

Se evidenciaron dos tipos de intenciones relacionadas con el uso del cuerpo y el movimiento en las prácticas educativas de los maestros y las maestras para potencializar el desarrollo integral de los infantes: por un lado, están las preparatorias o de primarización, que se reflejaron en las narrativas de las maestras, debido a que especificaron su propósito de formar, de manera efectiva, a los niños para su proceso educativo $y$, asimismo, para su futuro académico y laboral. Se encontró que estas intenciones probablemente están influenciadas por la necesidad que tienen los maestros y maestras de cumplir con los objetivos que el currículo escolar de la institución pide. Por otro lado, se identificaron las intenciones pedagógicas, es decir, aquellas que buscaron potencializar las funciones cognitivas. De esta forma, se detectó una carencia en las intenciones, pues estas van más dirigidas hacia el aprendizaje que hacía el desarollo integral de los infantes.

A partir de lo mencionado, se analiza que hay poca articulación con la política de la El de Colombia ya que, en la actualidad, el propósito de esta no es enseñar contenidos temáticos, como se realiza comúnmente en los colegios, sino implementar propuestas educativas que potencien el desarrollo integral de los niños (MEN, 2014c), y ofrecer al niño o niña condiciones favorables para su socialización, con el fin de valorar y desarrollar todas sus potencialidades, como lo mencionan Castro et al. (2015). Por lo tanto, se identificó que es necesario que los maestros y maestras fortalezcan el ejercicio de reflexión y de crítica ante el porqué de la realización de sus prácticas educativas e, igualmente, ante su propia comprensión del concepto del desarrollo.

Ahora bien, es claro que los primeros años de vida son una base determinante para el crecimiento de las personas, debido a que esta etapa se caracteriza por atravesar un proceso de constantes transformaciones cognitivas, físicas, afectivas y sociales, generando la necesidad de una atención integral (MEN, 2007). A partir de lo hallado en la presente investigación, nace una inquietud ante la mirada poco integral del desarrollo humano que el sistema educativo le está dando, ya que esto puede incidir en una formación incompleta que afecta el bienestar y el proceso de crecimiento de los niños y las niñas.

Para finalizar, es importante resaltar que, aunque se respondieron las preguntas de investigación plasmadas en este estudio, se presentan ciertas limitaciones relacionadas con la población estudiada, debido a que la muestra participante corresponde a tan solo una pequeña parte en relación con todo el sistema educativo de Colombia. Por tal razón, es 
importante invitar a colegas y a otros profesionales cercanos al tema a investigar sobre el desarrollo integral en consonancia con el cuerpo y el movimiento haciendo uso de una muestra más grande, para que así puedan confirmar o rectificar los resultados aquí plasmados.

\section{Referencias}

Aisenstein, Á.; Scharagrodsky, P. (2006). Tras las huellas de la educación física escolar Argentina: cuerpo, género y pedagogía: 1880-1950. Buenos Aires: Prometeo Libros.

Amar, J.; Abello, R.; Tirado, D. (2004). Desarrollo infantil y construcción del mundo social. (Marcos, A., ed.). Barranquilla: Ediciones Uninorte.

Barbero, J. I. (2005). La escolarización del cuerpo: reflexiones en torno a la levedad de los valores del capital "cuerpo" en educación física. Revista Iberoamericana de Educación, 39, 2551. Recuperado de: http://dialnet.unirioja.es/ servlet/articulo?codigo $=2007894$

Blanco, M. R. (2005). La educación de calidad para todos empieza en la primera infancia. Enfoques Educacionales, 7(1), 11-33.

Bolaños, G. (2006). Educación por medio del movimiento y la expresión corporal. San José, Costa Rica: Universidad Estatal a Distancia. DOI: https://doi.org/10.15517/revedu.v29i2.2248

Cabezas, H. (2005). Esquema corporal: una conducta básica para el aprendizaje del niño con autismo. Educación, 29(2), 207-215. DOI: https://doi.org/10.15517/revedu.v29i2.2248

Campo, L. A. (2010). Importancia del desarrollo motor en relación con los procesos evolutivos del lenguaje y la cognición en niños de 3 a 7 años de la ciudad de Barranquilla (Colombia). Salud Uninorte, 26(1), 65-76.

Cano, F. J.; Rodríguez, L.; García, J.; Antuña, M. A. (2005). Introducción a la psicología de la personalidad aplicada a las ciencias de la educación. Manual teórico. Sevilla: MAD-Eduforma.

Castro, J. A.; Bossio, A.; Rodríguez, H.; Villamizar, N. J. (2015). Análisis de la coherencia entre la política nacional de la educación inicial y el currículo operativo en primera infancia. Infancias Imágenes, 14(1), 112-124. DOI: https://doi. org/10.14483/udistrital.jour.infimg.2015.1.a08
Centro Internacional de Educación y Desarrollo Humano. (2001). Experiencias significantes de desarrollo infantil temprano en América Latina y Caribe. Bogotá: Unicef.

Cordero, T. (2004). Educación inicial en América Latina: situaciones y retos. Caso panameño. Educación, 28(1), 39-53.

Cruz de Oliveira, N. R. (2014). Corpo e movimento: olhar sobre práticas docentes e qualidade na educação infantil. Contrapontos, 14(1), 127 142. DOI: https://doi.org/10.14210/contrapontos.v14n1.p127-142

Fernández, M. J. (2010). EL libro de la estimulación para chicos de 0 a 36 meses. Buenos Aires: Albatros.

Gallego del Castillo, F. (2010). Esquema corporal y praxia: bases conceptuales. Sevilla: Wanceulen.

García, I.; Pérez, R.; Calvo, Á. (2013). Expresión corporal. Una práctica de intervención que permite encontrar un lenguaje propio mediante el estudio y la profundización del empleo del cuerpo. Retos. Nuevas tendencias en educación física, deporte y recreación, 23, 19-22.

Gil, P.; Contreras, O. R.; Gómez, I. (2008). Habilidades motrices en la infancia y su desarrollo desde una educación física animada. Revista Iberoamericana de Educación, 47, 71-96. Recuperado de: http://www.rieoei.org/rie47a04. $\underline{\mathrm{htm}}$

Grasso, A. (2005). Construyendo identidad corporal. La corporeidad escuchada. Buenos Aires: Novedades Educativas.

Hernández, R.; Fernández, C.; Baptista, P. (2010). Metodología de la investigación. México: McGraw-Hill.

Jaritonsky, P. (2001). Expresión corporal en el nivel inicial. Buenos Aires: Gobierno de la Ciudad Autónoma de Buenos Aires.

Justo, E. (2014). Desarrollo psicomotor en educación infantil. Bases para la intervención en psicomotricidad. Almería, España: Universidad de Almería.

Lení, V.; Wey, W. (2015). Movimiento y expresión corporal en educación infantil. Madrid: Narcea.

Ministerio de Educación Nacional (MEN). (2007). Bases sólidas para el desarrollo humano. Recuperado de: http://www.mineducacion.gov. co/1621/article-133793.html 
. (2014a). El arte en la educación inicial. Bogotá: MEN.

. (2014b). El juego en la educación inicial. Bogotá: MEN.

. (2014c). Sentido de la educación inicial. Bogotá: MEN.

Palau, E. (2004). Aspectos básicos del desarrollo infantil: la etapa de 0 a 6 años. Madrid: Grupo Planeta GBS.

Remorini, C. (2010). Crecer en movimiento. Abordaje etnográfico del desarrollo infantil en comunidades Mbya (Argentina). Revista Latinoamericana de Ciencias Sociales, Niñez y Juventud, 8(2), 961-980.

Robles, B. (2008). La infancia y la niñez en el sentido de identidad. Comentarios en torno a las etapas de la vida de Erik Erikson. Revista Mexicana de Pediatría, 75, 29-34.

Ruano, M. T.; Learreta, B.; Barriopedro, M. I. (2006). El valor de la emoción en expresión corporal desde las diferentes orientaciones. Kronos, 5(9), 24-33.

Sánchez, S.; Cañabete, D.; Montserrat, C.; Viscarro, I. (2014). Música, movimiento y danza: un enfoque integrador para la formación inicial del profesorado. Educatio Siglo XXI, 32(3), 145158. DOI: https://doi.org/10.6018/j/211021
Sarduy, Y. (2007). El análisis de información y las investigaciones cuantitativa y cualitativa. Revista Cubana de Salud Pública, 33(2), 1-11. DOI: https://doi.org/10.1590/ $\underline{\text { S0864-34662007000300020 }}$

Sarle, P.; Ivaldi, E.; Hernández, L. (2014). Arte, educación y primera infancia: sentidos y experiencias. Madrid: OEI.

Secretaría Distrital de Inclusión Social (SDIS); Universidad Pedagógica Nacional (UPN); Secretaría de Educación Distrital (SED). (2013). Lineamiento pedagógico y curricular para la educación inicial en el Distrito. Bogotá: Alcaldía Mayor de Bogotá.

Shoval, E.; Sharir, T.; Shulruf, B. (2014). The chaIlenge kindergarten model: Integrating bodymovement and scaffolding to keep the child in the center and make systematic progress in learning. Early Childhood Education Journal, 42, 279-286. DOI: https://doi.org/10.1007/ s10643-013-0609-2

Strauss, A.; Corbin, J. (2002). Bases de la investigación cualitativa. Técnicas y procedimientos para desarrollar la teoría fundamentada. Medellín: Universidad de Antioquia. 